\title{
Serologic testing of a panel of five antibodies in inflammatory bowel diseases: Diagnostic value and correlation with disease phenotype
}

\author{
ZHI-ZHI WANG ${ }^{1}$, KE SHI ${ }^{2}$ and JIE PENG ${ }^{1}$ \\ ${ }^{1}$ Department of Gastroenterology and ${ }^{2}$ Laboratory of the Department of Gerontology, \\ Xiangya Hospital, Central South University, Changsha, Hunan 410008, P.R. China
}

Received September 26, 2016; Accepted January 5, 2017

DOI: $10.3892 /$ br. 2017.860

\begin{abstract}
The aim of the present study was to evaluate the diagnostic value of five serological antibodies, perinuclear antineutrophilcytoplasmicantibody (pANCA), anti-Saccharomyces cerevisiae antibodies [ASCA; ASCA-immunoglobulin (IgG) and ASCA-IgA], Escherichia coli outer membrane porin $\mathrm{C}$ antibody (anti-OmpC) and CBir1 flagellin antibody for detection in inflammatory bowel diseases. Whether the antibody status correlated with the disease phenotype was also evaluated. Sera from 71 patients with Crohn's disease (CD), 41 patients with ulcerative colitis (UC), 78 patients with other gastrointestinal diseases and 31 healthy control subjects were investigated. Clinical data were gathered at the time of serum sampling and enzyme-linked immunosorbent assay was used to determine titers of the above mentioned five antibodies. The pANCA test exhibited a sensitivity of $53.7 \%$ for UC and the ASCA test had a sensitivity of $66.2 \%$ for CD. The prevalence of anti-OmpC was significantly higher in $\mathrm{CD}$ than in intestinal tuberculosis (TB), indicating that anti-OmpC may be a serologic marker distinguishing $\mathrm{CD}$ from $\mathrm{TB}$. The pANCA ${ }^{+} / \mathrm{ASCA}$ - exhibited the best specificity for differentiating between $\mathrm{CD}$ and UC. In UC, the presence of pANCA was greater in the patients with moderate to severe activity than in those with mild activity. ASCA was more positive in ileal CD. Furthermore, positive ASCA-IgG or anti-OmpC implied that
\end{abstract}

Correspondence to: Dr Jie Peng, Department of Gastroenterology, Xiangya Hospital, Central South University, 87 Xiangya Road, Changsha, Hunan 410008, P.R. China

E-mail: pengjie2014@csu.edu.cn

Dr Ke Shi, Laboratory of the Department of Gerontology, Xiangya Hospital, Central South University, 87 Xiangya Road, Changsha, Hunan 410008, P.R. China

E-mail: 137442165@qq.com

Key words: inflammatory bowel disease, Crohn's disease, ulcerative colitis, serological antibodies, perinuclear antineutrophil cytoplasmic antibody, anti-Saccharomyces cerevisiae antibodies, anti-outer membrane porin C, CBirl flagellin antibody complicated CD and pANCA was associated with colonic CD. Seropositivity of anti-CBirl was lowest in colonic CD.

\section{Introduction}

Inflammatory bowel disease (IBD) refers to a group of idiopathic inflammatory disorders of the gastrointestinal (GI) tract characterized by the manifestations of abdominal pain and diarrhea, the course of which is a chronic-recurrent process. Crohn's disease (CD) and ulcerative colitis (UC) are two types of IBD and exhibit overlapping and different clinical and pathological features (1). Its incidence in developing countries has gradually increased over the past five decades. The reported age-standardized incidence of IBD, CD and UC in China was 1.77-3.14 per $100,000,0.13-1.09$ per 100,000 and $1.45-2.05$ per 100,000, respectively (2-4). The estimated prevalence of UC and CD in China was 11.6 and 1.4 per 100,000 persons, respectively (5).

IBD is diagnosed by the comprehensive analysis of clinical findings, radiological imaging, invasive endoscopy and histopathological examination. Due to a lack of a gold standard, certain patients do not receive a definitive diagnosis using current diagnostic criteria, and an additional 5-15\% of chronic IBD cases cannot be classified as UC or CD, and are defined as indeterminate colitis (IC) or IBD unclassified (IBDU) (6). Furthermore, the pathogenesis of IBD remains unclear, although it is proposed to be caused by environmental effects and infection in genetically predisposed individuals, and mediated by immune mechanisms. Recently, the search for assistance with, or partial replacement of, diagnostic means and the elucidation of the pathogenesis have become the focus of IBD research, and serum antibody markers appear to be a good combination of these two aspects. Since antineutrophil cytoplasmic antibody (ANCA) and anti-Saccharomyces cerevisiae antibodies (ASCA) were first discovered in the 1990s, increasing numbers of serum antibodies have been identified, including Escherichia coli outer membrane porin C antibody (anti-OmpC) and antibody to CBirl flagellin (anti-CBirl), amongst others.

The majority of IBD serological studies are performed abroad, and the subjects are predominantly Caucasian. However, it has been shown that the prevalence of antibodies 
differs between locations or ethnic groups. Serological research in the clinical diagnosis of IBD in China has only recently begun, thus, there are few relevant reports and data. Furthermore, the research conclusions have often been inconsistent and it has been difficult to draw guidance that could be applied to all locations. The aim of the present study was to evaluate the clinical value of the abovementioned five serum antibodies (ANCA, ASCA-IgG and ASCA-IgA, anti-OmpC and anti-CBirl) in Chinese IBD patients, including their value for differentiating between IBD and non-IBD (N-IBD) GI diseases, UC and CD. In addition, their association with disease phenotypes (location, activity and complications) was investigated.

\section{Materials and methods}

Sample collection. A case group was developed consisting of CD and UC patients treated in the Department of Gastroenterology of Xiangya Hospital, Central South University (Changsha, China) between March 2015 and November 2015. Gender- and age-matched patients with non-IBD diseases (N-IBD) were defined as the disease control group, and gender- and age-matched healthy individuals from the Physical Examination Department of Xiangya Hospital served as the healthy control group. Clinical data (gender, age, disease duration, clinical manifestation, laboratory tests, endoscopic and histological examinations) were recorded when the serum sample was drawn. A total of $2 \mathrm{ml}$ venous blood was obtained from each subject (in the morning, fasted). All of the samples were spun at a speed of $1,000 \mathrm{x}$ g for $10 \mathrm{~min}$ within $2 \mathrm{~h}$ of collection, and the upper serum was collected and frozen at $-80^{\circ} \mathrm{C}$ until the assays were performed.

IBD case inclusion criteria included typical clinical manifestations, such as abdominal pain, diarrhea and purulent stools. The diagnosis of CD or UC was established by standard laboratory and radiological findings, and endoscopic criteria according to the 2010 World Gastroenterology Organization Practice Guidelines for IBD (1). CD and UC were subgrouped according to the Montreal classification (7).

The N-IBD group consisted of patients with non-IBD GI disorders, including other gastrointestinal diseases (OGID) and intestinal tuberculosis (TB). The patients with normal colonoscopy and pathology, and normal imaging were considered as the healthy control group. The exclusion criteria for all the subjects included acute and chronic infection of the GI tract other than TB, and a history of autoimmune diseases and cancer.

Written informed consent was obtained from all of the participants and the present study was approved by the Ethics Committees of Xiangya Hospital, Central South University.

Enzyme-linked immunosorbent assay (ELISA). All of the serum samples were analyzed using a standardized ELISA to detect the five antibodies, and the ELISA kits were obtained from Shen Yu Technology Co., Ltd. (Changsha, China). All of the specimens and kit reagents were restored to room temperature $\left(20-25^{\circ} \mathrm{C}\right)$ before use. Sera were diluted 1:10 in phosphate-buffered saline (PBS) and $100 \mu 1$ of the diluted sera was dispensed into the appropriate wells (2 wells per sample). For the reagent blank, $100 \mu$ l diluent was dispensed

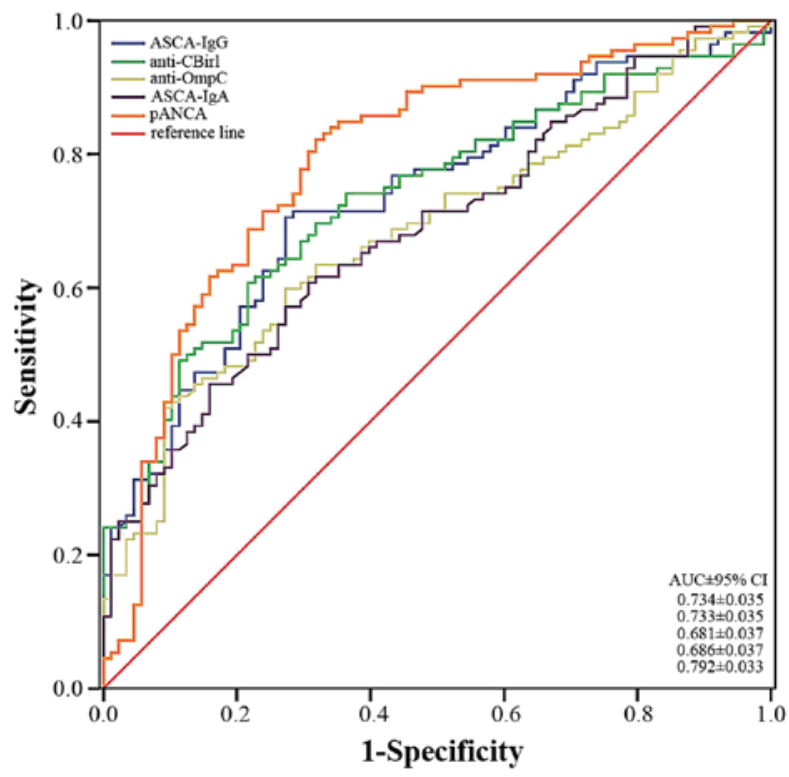

Figure 1. Receiver operating characteristic curves for discriminating between IBD and non-IBD patients. The AUC is shown for the five antibodies. IBD, inflammatory bowel disease; AUC, area under the curve; ASCA, anti-Saccharomyces cerevisiae antibody; IgG, immunoglobulin G; anti-CBir1, antibody to CBir1 flagellin; IgA, immunoglobulin A; pANCA, perinuclear antineutrophil cytoplasmic antibody.

in the $1 \mathrm{~A}$-well position. The samples were incubated for $30 \mathrm{~min}$ at room temperature, the liquid was removed from all of the wells, and the wells were washed three times in a PBS-Tween solution (Shen Yu Technology Co., Ltd.) followed by incubation, according to the manufacturer's instructions, with $100 \mu \mathrm{l}$ peroxidase-labeled goat anti-human IgG or IgA (Sigma-Aldrich; Merck KGaA, Darmstadt, Germany). Following the incubation for $30 \mathrm{~min}$ at room temperature, the enzyme conjugate was removed from all of the wells. The wells were then washed three times, and $100 \mu \mathrm{l}$ 3,3', 5,5'-tetramethylbenzidine substrate (Shen Yu Technology Co., Ltd.) was dispensed and incubated for $15 \mathrm{~min}$ at room temperature. A total of $100 \mu \mathrm{l}$ of the stop solution was then added, and the optical density (OD) of the reaction was read within $15 \mathrm{~min}$ at a wavelength of $450 \mathrm{~nm}$ using an ELISA reader. As qualitative ELISA assays were used, the cut-off values were determined according to the receiver operating characteristic (ROC) curve, and an OD greater than the cut-off value was considered positive. The ROC curves for the five antibodies are presented in Fig. 1. The area under the curve (AUC) for the IBD versus N-IBD with the five antibodies was $\geq 0.7$, indicating their ability to diagnose IBD. Perinuclear antineutrophil cytoplasmic antibody (pANCA) was clearly the most accurate marker for differentiating patients with IBD from patients with other diseases [AUC 0.792 and 95\% confidence interval $(\mathrm{CI}), \pm 0.033$ ). In addition, ASCA-IgG and anti-CBirl demonstrated good diagnostic accuracy (AUC $0.734 \pm 0.035$ and $0.733 \pm 0.035$, respectively).

Statistical analysis. Statistical analysis was performed using SPSS 19.0 (IBM SPSS, Armonk, NY, USA). The sensitivity, specificity, negative predictive value (NPV) and positive predictive value (PPV) of pANCA, ASCA (IgG and/or IgA), anti-OmpC, anti-CBirl and their different combinations were 
Table I. Clinical data of patients with CD and UC, and N-IBD and healthy control subjects.

\begin{tabular}{|c|c|c|c|c|}
\hline Characteristic & $\begin{array}{c}C D \\
n=71\end{array}$ & $\begin{array}{c}\mathrm{UC} \\
\mathrm{n}=41\end{array}$ & $\begin{array}{c}N-I B D^{a} \\
n=78\end{array}$ & $\begin{array}{l}\text { Healthy group } \\
n=31\end{array}$ \\
\hline Male/female & $49 / 22$ & $24 / 17$ & $44 / 34$ & $20 / 11$ \\
\hline Mean age (years) & $33.9 \pm 13.4$ & $46.1 \pm 13.7$ & $45.9 \pm 15.7$ & $42.1 \pm 13.9$ \\
\hline Range & $15-72$ & $17-72$ & $15-77$ & $15-70$ \\
\hline Disease duration (years) & $2.3 \pm 2.7$ & $2.9 \pm 3.6$ & & \\
\hline \multicolumn{5}{|l|}{ Disease location: UC, n (\%) } \\
\hline E1, Proctitis & & $6(14.6)$ & & \\
\hline E2, Left side & & $21(51.2)$ & & \\
\hline E3, Extensive & & $14(34.2)$ & & \\
\hline \multicolumn{5}{|l|}{ Severity of UC, n (\%) } \\
\hline S1, Mild & & & $13(31.7)$ & \\
\hline S2, Moderate & & & $19(46.3)$ & \\
\hline S3, Severe & & & $9(22.0)$ & \\
\hline \multicolumn{5}{|l|}{ Disease location: CD, n (\%) } \\
\hline L1, Terminal ileum & $31(43.6)$ & & & \\
\hline L2, Colon & $18(25.4)$ & & & \\
\hline L3, Ileocolon & $18(25.4)$ & & & \\
\hline L4, Upper GI & $4(5.6)$ & & & \\
\hline \multicolumn{5}{|l|}{ Clinical disease activity: CDAI, n (\%) } \\
\hline Mild & $15(21.1)$ & & & \\
\hline Moderate & $35(49.3)$ & & & \\
\hline Severe & $21(29.6)$ & & & \\
\hline \multicolumn{5}{|l|}{ Disease behavior: CD, n (\%) } \\
\hline B1, Non-stricturing, non-penetrating & $45(63.4)$ & & & \\
\hline B2, Stricturing & $22(31.0)$ & & & \\
\hline B3, Penetrating & $2(2.8)$ & & & \\
\hline $\mathrm{B} 2{ }^{+} \mathrm{B} 3$, Stricturing and penetrating & $2(2.8)$ & & & \\
\hline
\end{tabular}

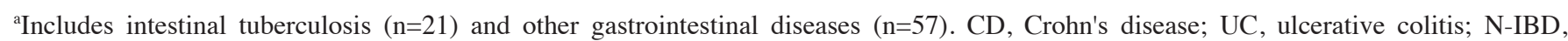
non-inflammatory bowel disease; CDAI, Crohn's Disease Activity Index.

determined for distinguishing between the UC, CD and control groups. For the data analyses, $\chi^{2}$ test or Fisher's exact test, as appropriate, were used to compare the frequency of positive antibodies between the study groups. The nonparametric Kruskal-Wallis test or Spearman's correlation assay was utilized to compare the median levels of antibody titers (the levels of the OD value) among different disease phenotypes. $\mathrm{P}<0.05$ was considered to indicate statistically significant differences.

\section{Results}

Demographics. A total of 112 unrelated IBD patients (CD, $\mathrm{n}=71$; UC, $\mathrm{n}=41$ ), 78 patients with $\mathrm{N}$-IBDs and 31 normal healthy individuals were enrolled in the present study. The $\mathrm{N}$-IBD group consisted of patients with chronic gastroenteritis $(\mathrm{n}=26)$, irritable bowel syndrome $(\mathrm{n}=5)$, functional dyspepsia $(n=5)$, GI polyps $(n=10)$, stromal tumor $(n=4)$, diverticulosis $(n=2)$, intestinal flora imbalance $(n=2)$, mixed hemorrhoids $(n=2)$, gastroesophageal reflux disease $(n=1)$ and intestinal TB $(n=21)$. The characteristics of the four populations are provided in Table I. No significant difference was identified in gender composition among each group ( $\mathrm{P}>0.05)$, whereas the $\mathrm{CD}$ group had a significantly younger mean age $(\mathrm{P}<0.05)$. According to the Montreal classification (7), UC patients were classified as follows: Proctitis (E1), left-sided colitis (E2), or pancolitis (E3), and the severity was classified as S0 (remission; none occurred because all of the patients enrolled in the present were inpatients), S1 (mild), S2 (moderate) and S3 (severe). The CD patients were subgrouped by disease behavior (B1, non-stricturing/nonpenetrating; B2, stricturing; and B3, penetrating) and disease location (L1, terminal ileum; L2, colon; L3, ileocolon; and L4, upper GI tract). According to the Crohn's Disease Activity Index (CDAI) (8), the clinical activity of $\mathrm{CD}$ patients was measured as mild, moderate or severe.

Diagnostic precision of a single antibody. The unique antibody patterns for the five antibodies are provided in Table II. ASCA-IgG was positive in $52.1 \%$ of CD patients compared with $31.7 \%$ of UC patients $(\mathrm{P}<0.05), 17.5 \%$ of OGID patients and only $6.5 \%$ of the healthy group. The 
Table II. Prevalence of five individual antibodies in each group.

\begin{tabular}{|c|c|c|c|c|c|}
\hline Antibody & $\mathrm{CD}(\mathrm{n}=71$ & $\mathrm{UC}(\mathrm{n}=41)$ & $\mathrm{TB}(\mathrm{n}=21)$ & OGID (n=57) & Healthy $(n=31)$ \\
\hline ASCA-IgG (\%) & $\mathrm{n} 37(52.1)^{\mathrm{a}, \mathrm{b}}$ & $13(31.7)$ & $8(38.1)$ & $10(17.5)$ & $2(6.5)$ \\
\hline ASCA-IgA (\%) & $\mathrm{n} 24(33.8)^{\mathrm{b}}$ & $7(17.1)$ & $3(14.3)$ & $5(8.8)$ & $0(0.0)$ \\
\hline pANCA $(\%)$ & $\mathrm{n} 15(21.1)^{\mathrm{b}}$ & $22(53.7)^{\mathrm{c}, \mathrm{d}}$ & $7(33.3)$ & $4(7.0)$ & $1(3.2)$ \\
\hline Anti-CBir1 (\%) & $\mathrm{n} 41(57.7)^{\mathrm{a}, \mathrm{b}}$ & $7(17.1)$ & $9(42.9)$ & $7(12.3)$ & $2(6.5)$ \\
\hline Anti-OmpC (\%) & $\mathrm{n} 36(50.7)^{\mathrm{a}, \mathrm{b}}$ & $13(31.7)$ & $4(19.0)^{\mathrm{e}}$ & $10(17.5)$ & $1(3.2)$ \\
\hline
\end{tabular}

${ }^{\mathrm{a}} \mathrm{P}<0.05$ vs. UC, healthy control subjects and OGID; ${ }^{\mathrm{b}} \mathrm{P}<0.05$ vs. healthy control subjects and $\mathrm{OGID} ;{ }^{\mathrm{c}} \mathrm{P}<0.05$ vs. $\mathrm{CD}$; ${ }^{\mathrm{P}}<0.05$ vs. healthy control subjects and OGID; ${ }^{\mathrm{P}}<0.05$ vs. CD. CD, Crohn's disease; UC, ulcerative colitis; TB, tuberculosis; OGID, other gastrointestinal disease; ASCA, anti-Saccharomyces cerevisiae antibody; Ig, immunoglobulin; pANCA, perinuclear antineutrophil cytoplasmic antibody; anti-CBir1, antibody to CBir1 flagellin; anti-OmpC, Escherichia coli outer membrane porin C.

Table III. Sensitivity, specificity, PPV and NPV of diagnosis for CD or UC.

\begin{tabular}{|c|c|c|c|c|c|}
\hline Antibody & Comparison & Sensitivity (\%) & Specificity (\%) & PPV (\%) & $\operatorname{NPV}(\%)$ \\
\hline ASCA-IgG & CD vs. control & 52.1 & 86.4 & 75.5 & 69.1 \\
\hline ASCA-IgA & CD vs. control & 33.8 & 94.3 & 82.8 & 63.8 \\
\hline pANCA & UC vs. control & 53.7 & 94.3 & 81.5 & 81.4 \\
\hline Anti-CBir1 & CD vs. control & 57.7 & 89.8 & 82.0 & 72.5 \\
\hline Anti-OmpC & CD vs. control & 50.7 & 87.5 & 76.6 & 68.8 \\
\hline Anti-OmpC & CD vs. TB & 50.7 & 81.0 & 90.0 & 32.7 \\
\hline
\end{tabular}

PPV, positive predictive value; NPV, negative predictive value; $\mathrm{CD}$, Crohn's disease; UC, ulcerative colitis; ASCA, anti-Saccharomyces cerevisiae antibody; Ig, immunoglobulin; pANCA, perinuclear antineutrophil cytoplasmic antibody; anti-CBir1, antibody to CBir1 flagellin; anti-OmpC, Escherichia coli outer membrane porin C antibody; TB, tuberculosis.

Table IV. Combination of ASCA-IgG, ASCA-IgA, anti-CBir1 and anti-OmpC in the diagnosis of CD.

\begin{tabular}{llcrr}
\hline Antibody & Comparison & $\begin{array}{c}\text { Sensitivity } \\
\%\end{array}$ & $\begin{array}{c}\text { Specificity } \\
\%\end{array}$ & $\begin{array}{c}\text { PPV } \\
\% \\
\%\end{array}$ \\
\hline ASCA-IgA/G & CD vs. control & 66.2 & 83 & 75.8 \\
Anti-CBir1 and ASCA & CD vs. control & 80.3 & 76.1 & 73.1 \\
Anti-OmpC and ASCA & CD vs. control & 85.9 & 73.9 & 72.6 \\
Anti-OmpC, anti-CBir1 and ASCA & CD vs. control & 91.5 & 69.3 & 70.7 \\
\hline
\end{tabular}

Anti-CBir1 and ASCA, anti-CBir1 and/or ASCA (ASCA-IgA/G) positive; anti-OmpC and ASCA, anti-OmpC and/or ASCA positive; antiOmpC and anti-CBir1 and ASCA, at least one antibody positive. ASCA, anti-Saccharomyces cerevisiae antibody; Ig, immunoglobulin; pANCA, perinuclear antineutrophil cytoplasmic antibody; anti-CBir1, antibody to CBir1 flagellin; anti-OmpC, Escherichia coli outer membrane porin C antibody; CD, Crohn's disease; PPV, positive predictive value; NPV, negative predictive value.

presence of ASCA-IgA in CD patients was $33.8 \%$, which was not significantly higher than that observed in the UC patients $(17.1 \%)$, although was markedly higher than those observed in the OGID $(8.8 \%)$ and healthy $(0 \%)$ groups. Of the CD patients with positive ASCA-IgG, 37.8\% exhibited positive ASCA-IgA. The prevalence of pANCA was significantly higher in the UC group (53.7\%) than in the CD, OGID and healthy control groups (21.1, 7.0 and $3.2 \%$, respectively). In addition, the prevalence of pANCA was significantly higher in the CD group than in the OGID and healthy control groups. Of the CD patients, 57.7 and $50.7 \%$ were positive for anit-CBirl and anti-OmpC, respectively, which were significantly higher than in the UC group (17.1 and $31.7 \%$ ), the OGID group (12.3 and 17.5\%) and the healthy control group (6.5 and 3.2\%). No significant difference was identified between the OGID group and the healthy group in all five antibodies. The prevalence of anti-OmpC was greater in the CD group than in the TB group, while the prevalence of the other four antibodies showed no significant difference between the CD, UC and the TB group. 
Table V. Diagnostic precision of different combinations of antibodies for inflammatory bowel disease.

\begin{tabular}{lcccc}
\hline Combination & $\begin{array}{c}\text { Sensitivity } \\
(\%)\end{array}$ & $\begin{array}{c}\text { Specificity } \\
(\%)\end{array}$ & $\begin{array}{c}\text { Positive predictive } \\
\text { value }(\%)\end{array}$ & $\begin{array}{c}\text { Negative predictive } \\
\text { value }(\%)\end{array}$ \\
\hline $\mathrm{pANCA}^{+} / \mathrm{ASCA}^{+}$ & 72.3 & 78.4 & 81.0 & 69.0 \\
$\mathrm{pANCA}^{+} / \mathrm{ASCA}^{+} / \mathrm{anti-CBir}{ }^{+}$ & 82.1 & 71.6 & 78.6 & 75.9 \\
$\mathrm{pANCA}^{+} / \mathrm{ASCA}^{+} / \mathrm{anti-OmpC}{ }^{+}$ & 85.7 & 69.3 & 78.0 & 79.2 \\
$\mathrm{pANCA}^{+} / \mathrm{ASCA}^{+} / \mathrm{anti-CBir1} /$ /anti-OmpC & 89.3 & 64.8 & 76.3 & 82.6 \\
\hline
\end{tabular}

$\mathrm{ASCA}^{+}$, ASCA-IgG and/or IgA positive. pANCA, perinuclear antineutrophil cytoplasmic antibody; ASCA, anti-Saccharomyces cerevisiae antibody; anti-CBir1, antibody to CBir1 flagellin; anti-OmpC, Escherichia coli outer membrane porin C antibody.

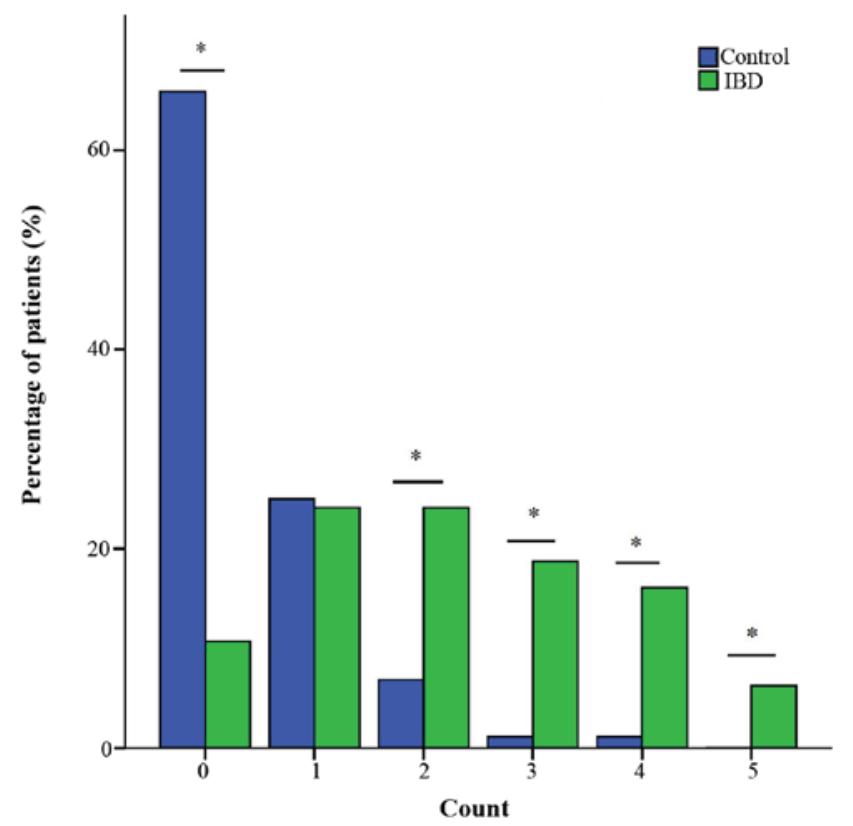

Figure 2. Number of positive antibodies: IBD vs. control. ${ }^{*} \mathrm{P}<0.05$. IBD, inflammatory bowel disease.

As there were no significant differences between the OGID group and healthy control group for all five antibodies, the OGID group and the healthy group were combined and served as the control group $(n=88)$, which was compared to the CD or UC groups. Sensitivity, specificity, PPV and NPV data for the five antibodies individually are provided in Table III. The sensitivity of ASCA-IgG and ASCA-IgA for CD was 52.1 and $33.8 \%$, respectively, and the specificity was 86.4 and $94.3 \%$, whereas PPV was 75.5 and $82.8 \%$, respectively. The sensitivity and specificity of pANCA for UC were 53.7 and $94.3 \%$, PPV was $81.5 \%$, and NPV was $81.4 \%$. When tested alone, the sensitivities of anti-CBirl and anti-OmpC for $\mathrm{CD}$ were 57.7 and $50.7 \%$, respectively and the specificities were 89.8 and $87.5 \%$, respectively. Due to the difference of anti-OmpC between CD and TB, the corresponding sensitivity, specificity, PPV, and NPV were calculated.

Diagnostic accuracy of combined antibodies. The diagnostic significance of the combined detection of four CD-associated antibodies is presented in Table IV. The sensitivity of

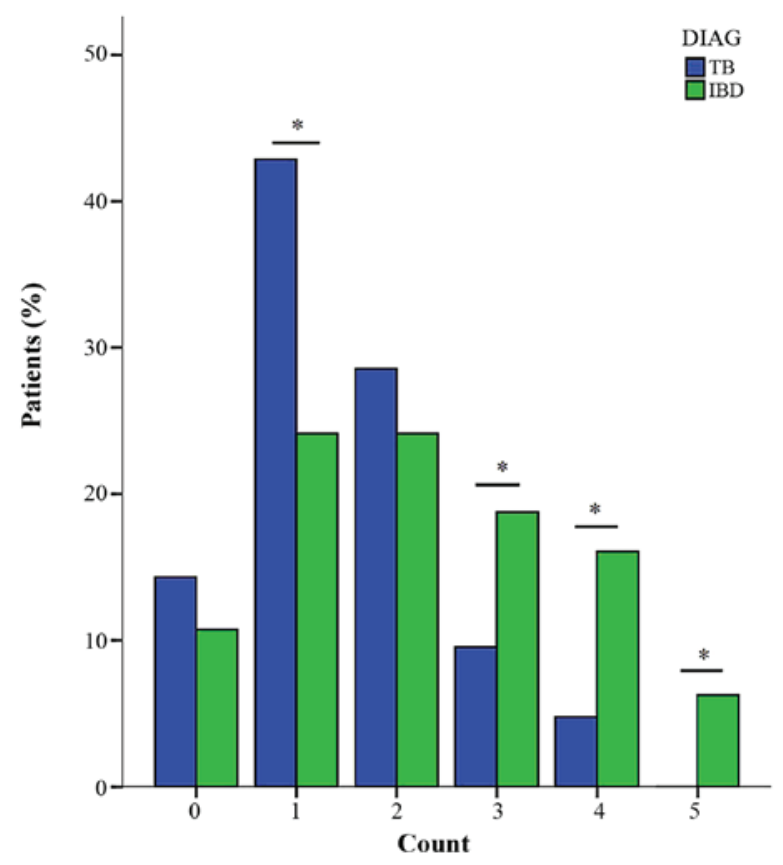

Figure 3. Number of positive antibodies: IBD vs. TB. ${ }^{*} \mathrm{P}<0.05$. IBD, inflammatory bowel disease; TB, tuberculosis.

ASCA-IgA for CD was low, but it increased to $66.2 \%$ when combined with ASCA-IgG. The specificity of ASCA-IgG/IgA was $83 \%$. When combined with anti-CBirl, the sensitivity of ASCA (ASCA-IgA and/or IgG) for CD increased to $80.3 \%$, and specificity decreased to $76.1 \%$. The combination of anti-OmpC and ASCA had a sensitivity of $85.9 \%$ for CD, and the specificity was $73.9 \%$. If a positive screening test was defined as the presence of at least one positive antibody, then the three-antibody panel (ASCA, anti-CBir1 and anti-OmpC) had an overall sensitivity of $91.5 \%$ for $\mathrm{CD}$, with a specificity of $69.3 \%$. Therefore, it was concluded that the diagnostic efficiency of combining the detection of anti-OmpC and ASCA was the highest.

The diagnostic precision of the combined testing of ASCA and pANCA, with or without anti-CBirl and/or anti-OmpC, in IBD (CD and UC, $n=112$ ) is presented in Table V. When compared with the control group (OGID and healthy group combined), $\mathrm{pANCA}^{+} / \mathrm{ASCA}^{+}$had a sensitivity of $72.3 \%$ and a specificity of $78.4 \%$ for IBD. However, the addition of anti-CBir1 improved the sensitivity to $82.1 \%$, while it slightly 
Table VI. ASCA and pANCA for single or combined differential diagnosis of CD and UC.

\begin{tabular}{llcccc}
\hline Comparison & Antibody & Sensitivity (\%) & Specificity (\%) & PPV (\%) & NPV (\%) \\
\hline CD vs. UC & ASCA $^{+}$ & 66.2 & 58.5 & 73.4 & 50 \\
& ASCA $^{+}$/pANCA & 50.7 & 80.5 & 81.8 & 51.5 \\
UC vs. CD & pANCA $^{+}$ & 53.7 & 78.9 & 59.5 & 74.7 \\
& pANCA $^{+} /$ASCA $^{-}$ & 31.7 & 94.4 & 76.5 & 70.5 \\
\hline
\end{tabular}

ASCA-, ASCA-IgG and -IgA (each are negative). ASCA, anti-Saccharomyces cerevisiae antibody; pANCA, perinuclear antineutrophil cytoplasmic antibody; $\mathrm{CD}$, Crohn's disease; UC, ulcerative colitis.

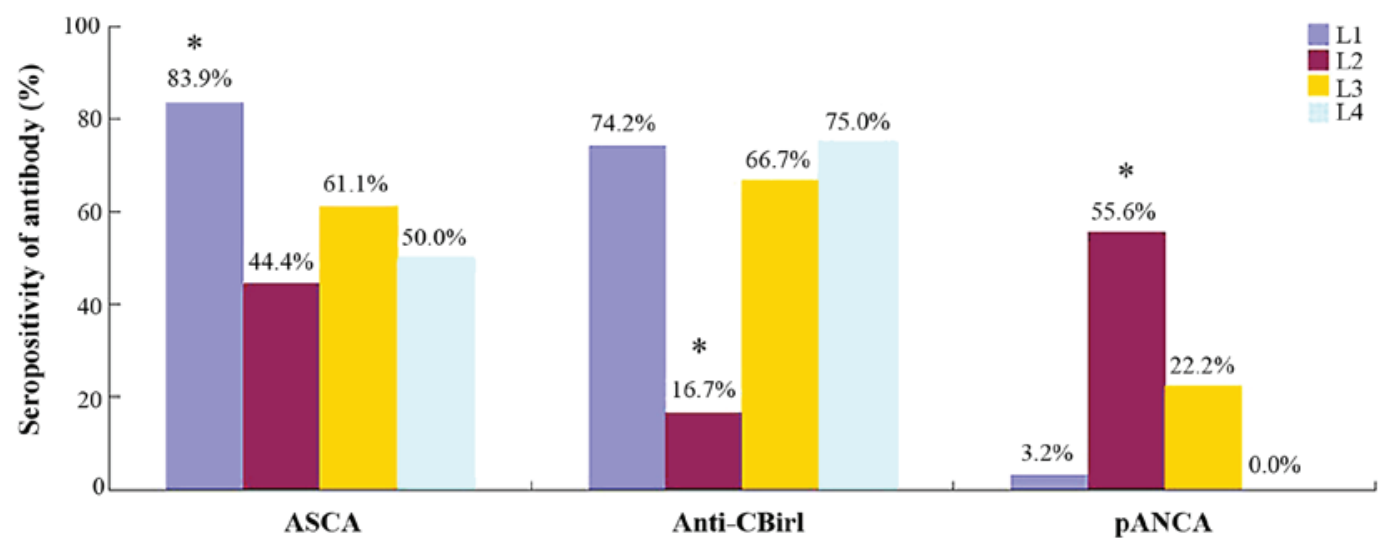

Figure 4. Seropositivity of antibodies in different Crohn's disease locations. L1, terminal ileum; L2, colon; L3, ileocolon; L4, upper GI tract. "P<0.05. ASCA, anti-Saccharomyces cerevisiae antibody; anti-CBir1, antibody to CBir1 flagellin; pANCA, perinuclear antineutrophil cytoplasmic antibody.

decreased the specificity to $71.6 \%$. However, after adding anti-OmpC, the sensitivity of $\mathrm{pANCA}^{+} / \mathrm{ASCA}^{+}$for IBD was $85.7 \%$, with a specificity of $69.3 \%$. Finally, $89.3 \%$ of IBD patients were positive for $\geq 1$ of the five antibodies, although the specificity and PPV decreased.

The number of positive antibodies in the five-antibody panel between IBD and the control group (Fig. 2), and between the IBD and the TB groups (Fig. 3) was compared. This finding indicated that the control group was more likely to have no positive antibody, and there was no significant difference between IBD and the control group when there was just one positive antibody. However, the percentage of patients that had $\geq 2$ positive antibodies in the IBD group was significantly higher. As demonstrated in Fig. 3, when the number was 0 or 2, no difference between IBD and TB was identified, whereas the TB group was more likely to have 1 positive antibody, and the IBD group was more likely to have $\geq 3$ antibodies.

Serologic antibodies in the differential diagnosis of $C D$ and UC. From the abovementioned results (Table II), it was identified that ASCA and PANCA were unique for CD and UC, respectively; however, ASCA was positive in $41.5 \%$ of UC patients and pANCA was positive in $21.1 \%$ of CD patients, which decreased their ability to differentiate between patients with UC and $\mathrm{CD}$. Therefore, the diagnostic accuracy of $\mathrm{ASCA}^{+} / \mathrm{pANCA}^{-}$and $\mathrm{pANCA}^{+} / \mathrm{ASCA}^{-}$in the differential diagnosis of $\mathrm{CD}$ and $\mathrm{UC}$ was evaluated, and the results are presented in Table VI. The sensitivity of $\mathrm{ASCA}^{+} / \mathrm{pANCA}^{-}$for distinguishing $\mathrm{CD}$ and $\mathrm{UC}$ was $50.7 \%$, and the specificity was $80.5 \%$. While the combination of $\mathrm{pANCA}^{+} / \mathrm{ASCA}^{-}$had a specificity of $94.4 \%$ for differentiating between UC and CD.

The importance of newly identified antibodies was noted by the observation that ASCA-negative $\mathrm{CD}$ patients may be positive for anti-CBirl and anti-OmpC. That result indicated that the two differentiated between the ASCA-negative CD patients and the control subjects. In addition, anti-OmpC had a differential value between $\mathrm{CD}$ and $\mathrm{UC}$, whereas anti-CBirl had no such value $(\mathrm{P}=0.112)$. However, neither anti-CBirl nor anti-OmpC was able to distinguish pANCA-positive $\mathrm{CD}$ from UC.

Correlation between antibodies and disease phenotype. Higher ASCA seropositivity was identified in CD patients with ileal lesions (L1) compared to patients with colonic (L2) disease, ileo-colonic (L3) disease or upper GI tract involvement (L4) (83.9\% vs. $44.4,61.1$ or $50 \%$, respectively; $\mathrm{P}<0.05)$. The ASCA seropositivity was not significantly different in the L2, L3, and L4 groups ( $>>0.05)$. Significantly higher ASCA-IgG prevalence was observed in patients with complicated (stricturing, penetrating or lesion of the anus) disease, compared with patients with non-complicated (B1) phenotype (75 vs. $33.3 \%$; $\mathrm{P}=0.001$ ). However, no significant difference was identified in the prevalence of ASCA-IgA between complicated diseases and simple diseases. According to ASCA titers in ASCA-IgG-positive CD, no significant difference among location subgroups or disease behaviors was identified. Furthermore, no correlation was observed between the disease 


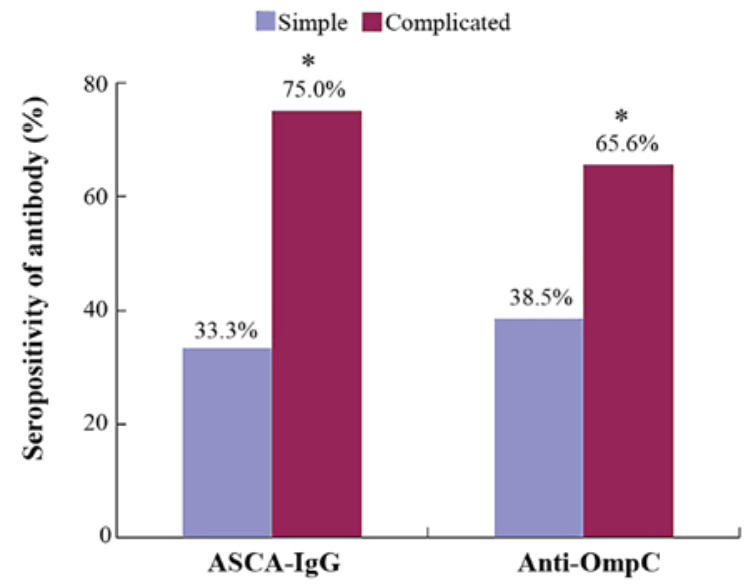

Figure 5. Seropositivity of antibodies in different Crohn's disease behaviors. ${ }^{*} \mathrm{P}<0.05$. ASCA, anti-Saccharomyces cerevisiae antibody; IgG, immunoglobulin G; anti-OmpC, Escherichia coli outer membrane porin C antibody.

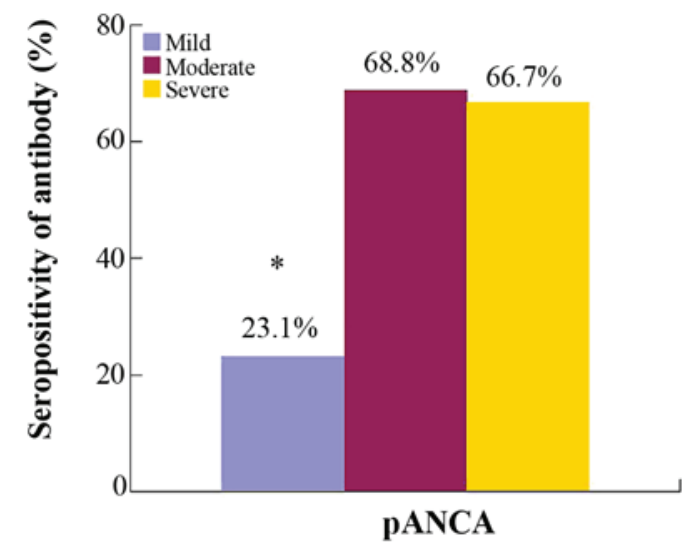

Figure 6. Prevalence of pANCA in different UC severities. "P<0.05. pANCA, perinuclear antineutrophil cytoplasmic antibody.

severity and ASCA seropositivity in the patients in the current study.

pANCA is present with a significantly higher frequency in CD patients with only colonic disease (L2, 55.6\%) compared with patients with ileal lesions (L1, 3.2\%), ileocolonic lesions (L3, 22.2\%) or upper GI tract involvement (L4,0\%); no significant difference was found among the latter three subtypes.

The prevalence of anti-CBirl was different among distinct locations of $\mathrm{CD}$ and multiple comparisons showed that those patients with colonic disease had lower seropositivity compared with patients with the other three phenotypes; although there was no significant difference among the other three subtypes. In $\mathrm{CD}$ with positive anti-CBirl, the anti-CBirl titers were not significantly different among the different location phenotypes. There was no correlation between the presence of anti-CBirl and disease behavior or activity. Qualitative anti-OmpC was not significantly different among the CD locations, but it was more common in the patients with complications than in those with pure inflammation. In $\mathrm{CD}$ with positive anti-OmpC, there was no positive correlation between anti-OmpC titers and complications. Similar to anti-CBirl, anti-OmpC was not associated with $\mathrm{CD}$ activity. The correlation between antibodies and CD phenotype are shown in Figs. 4 and 5.

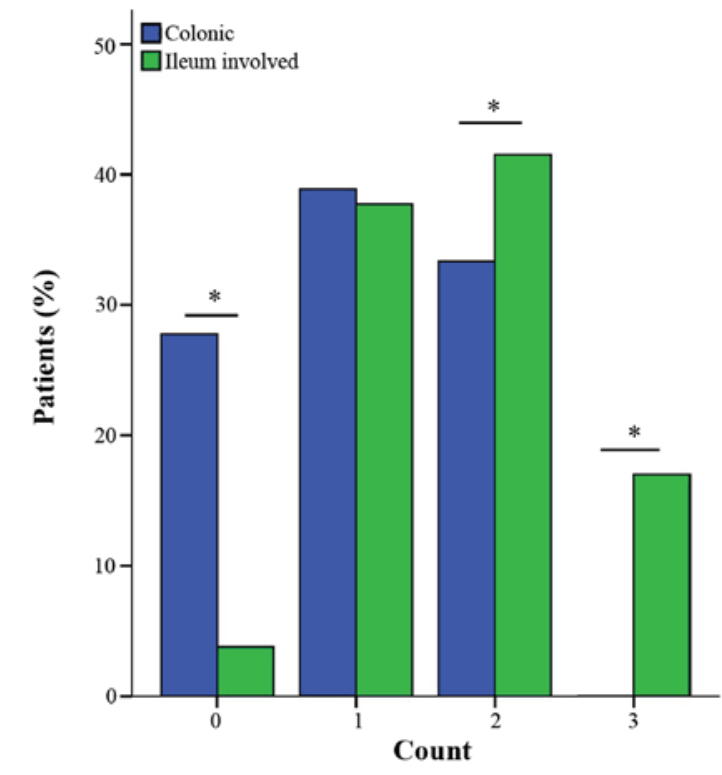

Figure 7. Number of positive antibodies in different Crohn's disease locations. ${ }^{*} \mathrm{P}<0.05$.

In UC patients, the presence of pANCA was not significantly different among the disease locations. The result of multiple comparison indicated that the patients with moderate and severe disease (S2 and S3) had significantly higher pANCA seropositivity than those with mild (S1) disease, whereas the two former were compared, and no difference was observed (Fig. 6). However, in UC with positive pANCA expression, the antibody titers of S2 and S3 were not higher than in S1.

The results of the correlation analysis demonstrated no correlation between IBD disease location, activity and complications. The Kruskal-Wallis test indicated the titers of ASCA, anti-CBirl and anti-OmpC were not correlated with the duration of $\mathrm{CD}$ and the titer of pANCA was not correlated with the duration of UC.

The determination of the disease phenotype by the combined test of antibodies was evaluated (Fig. 7). When anti-OmpC, anti-CBirl and ASCA were combined and all three antibodies were negative, the ratio of colonic $\mathrm{CD}$ was significantly higher than $\mathrm{CD}$ with ileum involvement. When only one antibody was positive, there was no difference between the two. When there were 2 or more antibodies positive, the ratio of $\mathrm{CD}$ with ileum involvement was significantly higher than colonic CD. However, no correlation between disease behavior and the number of positive antibodies was observed in the patients.

\section{Discussion}

ASCA and pANCA were the earliest identified serological antibodies correlated with IBD, and the studies date back to the 1990s. ASCA are targeted at the phosphopeptidomannan of the cell wall of Saccharomyces cerevisiae (9), including ASCA-IgG and ASCA-IgA. Previous studies have shownthat ASCA was detected in more CD patients (39-70\%) and their healthy relatives (25-20\%) than in healthy individuals without family history and in UC patients $(0-5 \%$ and $10-15 \%$, respectively) (10). ANCAs are autoanibodies directed against 
the cytosolic components of neutrophil granules and are represented by three main staining patterns as follows: Cytoplasmic granular, perinuclear and atypical. ANCAs were initially described in primary vasculitis, such as Wegener granulomatous, and the correlation between pANCA and UC was first established in 1990 (11). Studies have found that the prevalence of pANCA was 50-70\% in UC patients, $6-20 \%$ in $\mathrm{CD}$ patients and only $0-2.5 \%$ in healthy individuals, although the prevalence of pANCA in the healthy relatives of UC was not identified to be higher than that in ordinary people (10).

In the present study, the seroprevalence of ASCA-IgG in $\mathrm{CD}$ was $52.1 \%$, which was significantly higher than that in UC, OGIDs and the healthy group, and the seroprevalence of ASCA-IgA in CD was also significantly higher than in the two control groups. Similarly, 53.7\% of UC patients are seropositive for pANCA, significantly higher than in CD and the control groups. These results were consistent with studies that have been performed abroad $(10,12)$ and in domestic research $(13,14)$.

Anti-CBirl and anti-OmpC are newly identified bacterial antigen antibodies that target flagellin CBirl and E. coli outer membrane poreC, respectively. According to previous studies, the seropositivity of anti-CBirl in CD, UC and healthy control subjects was 50-57, 6-16 and 8-15\%, respectively (15). The antigen proteins of anti-OmpC were originally proposed to cross-react with pANCA, as the expression level of the IgG antibody in UC patients increased more than in healthy individuals (16). However, later experiments demonstrated that the IgA of anti-OmpC was more common in CD patients (17), and therefore $\operatorname{IgA}$ is generally detected. In a previous study, the seroprevalence of anti-OmpC ranged from $5-11 \%$ in UC patients, $20-55 \%$ of $\mathrm{CD}$ patients were seropositive for anti-OmpC and the prevalence in healthy control subjects was only 5\% (17). Similar to the above-mentioned studies, the present study showed that the seroprevalence of anti-CBirl and anti-OmpC in CD were significantly higher than that in $\mathrm{UC}$, OGIDs or healthy individuals. When tested independently, anti-CBirl and anti-OmpC had limited sensitivity, although their specificity was good. In addition, the sensitivity, specificity, and positive and negative predictive value of anti-CBirl were all higher than anti-OmpC. Therefore, it seemed that the diagnostic value of anti-CBir1 was better than anti-OmpC; however, to the best of our knowledge, there is no study comparing the two.

Regarding the differential diagnosis of intestinal TB and IBD in the present study, four antibodies had no differential ability except for anti-OmpC. Similarly, Makharia et al (18) found that ASCA or pANCA could not distinguish between TB and IBD; however, studies comparing the expression of anti-CBirl or anti-OmpC between IBD and TB are limited. In the present study, the PPV of anti-OmpC differentiating between CD and TB was as high as $90 \%$, and it may be used as a marker for the differential diagnosis of TB and CD; however, this requires further confirmation.

A combined test for ASCA-IgG and IgA may increase the diagnostic accuracy for $\mathrm{CD}$, which is consistent with a previous report (19). The addition of anti-CBirl increased the sensitivity of ASCA (ASCA-IgA and/or IgG) for CD from 66.2 to $80.3 \%$ with the specificity slightly decreasing (from 83 to $76.1 \%$ ); the sensitivity increased to $85.9 \%$ when joined with
anti-OmpC in ASCA and the specificity remained at $73.9 \%$. Our results were comparable with the conclusion of a study by Zholudev et al (20). When the four above-mentioned antibodies were detected, the sensitivity for CD was $91.5 \%$, but the specificity significantly decreased. The combined test of pANCA and ASCA had a higher sensitivity for IBD than that of any of the antibodies along, and the addition of anti-CBirl improved the sensitivity of this combination to $82.1 \%$, with a specificity of $71.6 \%$. The addition of anti-OmpC increased the sensitivity of the pANCA and ASCA combination to $85.7 \%$, with a specificity of $69.3 \%$. It may be concluded that the detection of an individual antibody has a limited sensitivity and a high specificity, which is not suitable for screening IBD in patients with GI symptoms, but rather is suitable as an adjunctive tool for patients in whom an endoscopic examination does not provide a certain diagnosis. The combined detection of antibodies improves diagnostic sensitivity and slightly decreases specificity, which may serve as a non-invasive screening tool. In particular, for the novel serological antibodies, the diagnostic value will be greater in the combined test than when tested independently.

Previous data have indicated that the higher the number of positive antibodies, the more possible the diagnosis for IBD (17). The present study also confirmed that when there were $\geq 2$ positive antibodies, IBD patients could be distinguished from OGIDs and healthy individuals. Furthermore, an increased percentage of IBD patients with an increasing diversity of the immune response was demonstrated, with the highest odds in patients that were positive for all five antibodies. Additionally, patients positive for $\geq 3$ antibodies were more likely to be diagnosed as IBD rather than TB, although the seropositivity of a single antibody between IBD and TB was similar.

Combined testing rather than evaluating individual antibodies is more useful in identifying IBD subtypes. The $\mathrm{ASCA}^{+} / \mathrm{pANCA} \mathrm{A}^{-}$profile had the best combined sensitivity and specificity for distinguishing CD from UC at 50.7 and $80.5 \%$, respectively. The reverse profile of $\mathrm{pANCA}^{+} / \mathrm{ASCA}$ - was most specific for differentiating $\mathrm{UC}$ from $\mathrm{CD}$, with a specificity of $94.4 \%$. These results are also consistent with a previous report (21).

The novel antibodies, anti-CBirl and anti-OmpC, may help to diagnose ASCA-negative $\mathrm{CD}$, and independent from ASCA, anti-OmpC may allow differentiation between ASCA-negative $\mathrm{CD}$ patients and ASCA-negative UC patients. This finding was consistent with the study by Joossens et al (17), although it was different from the conclusion that anti-CBirl or anti-OmpC could differentiate pANCA-positive UC from pANCA-positive $\mathrm{CD}$ (which was not established in the current study). However, other studies also confirmed that anti-CBirl was able to differentiate between pANCA-positive UC and CD (15). This discrepancy may result from differences regarding the pathogenesis between Chinese and Western individuals.

ASCA-IgG and IgA are associated with the disease location of $\mathrm{CD}$, their seropositivity were highest in ileal $\mathrm{CD}$. The proportion of ASCA-IgG-positive CD patients was significantly higher in complicated diseases (stenosis, perforation and anal diseases) compared with patients with uncomplicated diseases. These results have been proven by previous studies $(19,22)$. However, in ASCA-positive CD patients, the 
correlation between ASCA titers and disease locations or disease behavior was not identified in the present study. It was known that the prevalence of ASCA was not associated with CD severity, and the present study drew the same conclusion. Data showed that pANCA was associated with UC-like $\mathrm{CD}(13,23)$, and the current study found that the proportion of pANCA-positives was higher in colonic CD patients than in the other subgroups.

Anti-CBirl was the first bacterial antigen antibody that could induce colitis in rat models, which increased the detection of small-bowel disease, UC-like disease, and complicated disease, such as fibrostenosis or internal-penetrating disease (24). In the present study, qualitative anti-CBir1 was negatively associated with the colonic CD population; however, the quantitative level of anti-CBirl was found to be unassociated with disease locations in anti-CBirl-positive CD. Unlike certain studies that were conducted on a western population, anti-CBirl expression was not identified to be associated with $\mathrm{CD}$ complications in the current patients. Previous studies demonstrated that anti-OmpC was not associated with CD locations (23), and the present result was consistent with this finding. Furthermore, it was found that anti-OmpC expression was associated with $\mathrm{CD}$ behavior and had a higher prevalence in complicated CD. In addition, there was an association between anti-OmpC titer level and disease behaviors. Previous data showed that the presence of ASCA in CD patients was independent of disease activity and duration (19). In the present study, ASCA, anti-CBirl and anti-OmpC were independent of $\mathrm{CD}$ activity and the disease course.

Many foreign studies demonstrated that pANCA was not associated with the UC phenotype $(23,25)$. However, it has been shown in Chinese UC patients that pANCA was more frequent with extensive disease (26) and with active disease (27). In the present study, the prevalence of pANCA was significantly higher in moderate to severe UC than in mild UC, but it was unrelated with UC extension. In pANCA-positive UC, the titer of pANCA did not increase as the disease activity increased.

The results of the combined test of three antibodies (anti-OmpC, anti-CBir1 and ASCA) indicated that when the number of positive antibodies was $\geq 2$, the $C D$ patients were more likely to have ileum involvement. The above result was consistent with previous review articles (23). Furthermore, no association between the quantity of antibody and the disease behavior was identified in the present study.

In the present study, up to five antibodies were detected, alone and in combination, for the diagnosis of IBD. Their correlation with disease phenotype was analyzed in depth. To the best of our knowledge, these results are the first to indicate that anti-OmpC may potentially be the antibody that differentiates between $\mathrm{CD}$ and $\mathrm{TB}$. The subjects of the current study were primarily Chinese individuals in a limited area, which was somewhat representative. However, there were also certain limitations. The duration of the study was short and the number of cases was correspondingly small rather than a large cohort of IBD patients. A larger sample size and a more diverse population are required in order that the conclusions are more applicable. Previous studies have shown that serum antibody markers predicted the occurrence and progress of $\operatorname{IBD}(28,29)$.
The present study was a retrospective, non-prospective study, the association between the antibodies and the progress of diseases were not tracked, and the prediction of antibodies on the treatment response was not examined. Therefore, follow-up experimental studies are required in future.

\section{References}

1. Bernstein CN, Fried M, Krabshuis JH, Cohen H, Eliakim R, Fedail S, Gearry R, Goh KL, Hamid S, Khan AG, et al: World gastroenterology organization practice guidelines for the diagnosis and management of IBD in 2010. Inflamm Bowel Dis 16: $112-124,2010$.

2. Zeng Z, Zhu Z, Yang Y, Ruan W, Peng X, Su Y, Peng L, Chen J, Yin Q, Zhao C, et al: Incidence and clinical characteristics of inflammatory bowel disease in a developed region of Guangdong Province, China: A prospective population-based study. J Gastroenterol Hepatol 28: 1148-1153, 2013.

3. Zhao J, Ng SC, Lei Y, Yi F, Li J, Yu L, Zou K, Dan Z, Dai M, Ding Y, et al: First prospective, population-based inflammatory bowel disease incidence study in mainland of China: The emergence of 'western' disease. Inflamm Bowel Dis 19: 1839-1845, 2013

4. Yang H, Li Y, Wu W, Sun Q, Zhang Y, Zhao W, Lv H, Xia Q, $\mathrm{Hu}$ P, Li H and Qian J: The incidence of inflammatory bowel disease in Northern China: A prospective population-based study. PLoS One 9: e101296, 2014

5. Ye L, Cao Q and Cheng J: Review of inflammatory bowel disease in China. Sci World J 2013: 296470, 2013.

6. Arai R: Serologic markers: Impact on early diagnosis and disease stratification in inflammatory bowel disease. Postgrad Med 122: 177-185, 2010.

7. Satsangi J, Silverberg MS, Vermeire S and Colombel JF: The Montreal classification of inflammatory bowel disease: Controversies, consensus, and implications. Gut 55: 749-753, 2006.

8. Best WR, Becktel JM, Singleton JW and Kern F Jr: Development of a Crohn's disease activity index. National cooperative Crohn's disease study. Gastroenterology 70: 439-444, 1976.

9. Main J, McKenzie H, Yeaman GR, Kerr MA, Robson D, Pennington CR and Parratt D: Antibody to Saccharomyces cerevisiae (bakers' yeast) in Crohn's disease. BMJ 297: 1105-1106, 1988.

10. Peyrin-Biroulet L, Standaert-Vitse A, Branche J and Chamaillard M: IBD serological panels: Facts and perspectives. Inflamm Bowel Dis 13: 1561-1566, 2007.

11. Rump JA, Schölmerich J, Gross V, Roth M, Helfesrieder R, Rautmann A, Lüdemann J, Gross WL and Peter HH: A new type of perinuclear anti-neutrophil cytoplasmic antibody (p-ANCA) in active ulcerative colitis but not in Crohn's disease. Immunobiology 181: 406-413, 1990.

12. Kim BG, Kim YS, Kim JS, Jung HC and Song IS: Diagnostic role of anti-Saccharomyces cerevisiae mannan antibodies combined with antineutrophil cytoplasmic antibodies in patients with inflammatory bowel disease. Dis Colon Rectum 45: 1062-1069, 2002.

13. Zhou F, Xia B, Wang F, Shrestha UK, Chen M, Wang H, Shi X, Chen $\mathrm{Z}$ and $\mathrm{Li}$ J: The prevalence and diagnostic value of perinuclear antineutrophil cytoplasmic antibodies and anti-Saccharomyces cerevisiae antibodies in patients with inflammatory bowel disease in mainland China. Clin Chim Acta 411: 1461-1465, 2010.

14. Lawrance IC, Murray K, Hall A, Sung JJ and Leong R: A prospective comparative study of ASCA and pANCA in Chinese and caucasian IBD patients. Am J Gastroenterol 99: 2186-2194, 2004.

15. Papp $M$ and Lakatos PL: Serological studies in inflammatory bowel disease: How important are they? Curr Opin Gastroenterol 30: 359-364, 2014.

16. Landers CJ, Cohavy O, Misra R, Yang H, Lin YC, Braun J and Targan SR: Selected loss of tolerance evidenced by Crohn's disease-associated immune responses to auto- and microbial antigens. Gastroenterology 123: 689-699, 2002.

17. Joossens S, Reinisch W, Vermeire S, Sendid B, Poulain D, Peeters M, Geboes K, Bossuyt X, Vandewalle P,Oberhuber G, et al: The value of serologic markers in indeterminate colitis: A prospective follow-up study. Gastroenterology 122: 1242-1247, 2002 . 
18. Makharia GK, Sachdev V, Gupta R, Lal S and Pandey RM: Anti-Saccharomyces cerevisiae antibody does not differentiate between Crohn's disease and intestinal tuberculosis. Dig Dis Sci 52: 33-39, 2007.

19. Gologan S, Iacob R, Preda C, Vadan R, Cotruta B, Catuneanu M, Iacob S, Constantinescu I, Gheorghe L, Iobagiu S, et al: Higher titers of anti-Saccharomyces cerevisiae antibodies IgA and IgG are associated with more aggressive phenotypes in Romanian patients with Crohn's disease. J Gastrointestin Liver Dis 21: 39-44, 2012.

20. Zholudev A, Zurakowski D, Young W, Leichtner A and Bousvaros A: Serologic testing with ANCA, ASCA, and anti-OmpC in children and young adults with Crohn's disease and ulcerative colitis: Diagnostic value and correlation with disease phenotype. Am J Gastroenterol 99: 2235-2241, 2004.

21. Reese GE, Constantinides VA, Simillis C, Darzi AW, Orchard TR, Fazio VW and Tekkis PP: Diagnostic precision of anti-Saccharomyces cerevisiae antibodies and perinuclear antineutrophil cytoplasmic antibodies in inflammatory bowel disease. Am J Gastroenterol 101: 2410-2422, 2006.

22. Zhang Z, Li C, Zhao X, Lv C, He Q, Lei S, Guo Y and Zhi F: Anti-Saccharomyces cerevisiae antibodies associate with phenotypes and higher risk for surgery in Crohn's disease: A meta-analysis. Dig Dis Sci 57: 2944-2954, 2012.

23. Prideaux L, De Cruz P, Ng SC and Kamm MA: Serological antibodies in inflammatory bowel disease: A systematic review. Inflamm Bowel Dis 18: 1340-1355, 2012.
24. Targan SR, Landers CJ, Yang H, Lodes MJ, Cong Y, Papadakis KA, Vasiliauskas E, Elson CO and Hershberg RM: Antibodies to CBirl flagellin define a unique response that is associated independently with complicated Crohn's disease. Gastroenterology 128: 2020-2028, 2005.

25. Kuna AT: Serological markers of inflammatory bowel disease. Biochem Med (Zagreb) 23: 28-42, 2013.

26. Cioffi M, Rosa AD, Serao R, Picone I and Vietri MT: Laboratory markers in ulcerative colitis: Current insights and future advances. World J Gastrointest Pathophysiol 6: 13-22, 2015.

27. Wang X and Dong E: Anti-neutrophil cytoplasmic antibodies in patients with inflammatory bowel diseases. Chin J Integr Med 3: 15-18, 2000 (In Chinese).

28. Israeli E, Grotto I, Gilburd B, Balicer RD, Goldin E, Wiik A and Shoenfeld Y: Anti-Saccharomyces cerevisiae and antineutrophil cytoplasmic antibodies as predictors of inflammatory bowel disease. Gut 54: 1232-1236, 2005.

29. Devlin SM and Dubinsky MC: Determination of serologic and genetic markers aid in the determination of the clinical course and severity of patients with IBD. Inflamm. Bowel Dis 14: $125-128,132-133,2008$ 